\title{
Density of Rational Points on a Certain Smooth Bihomogeneous Threefold
}

\section{Pierre Le Boudec}

\author{
EPFL SB MATHGEOM TAN, MA C3 604 (Bâtiment MA), Station 8, \\ CH-1015 Lausanne, Switzerland
}

Correspondence to be sent to: pierre.leboudec@epfl.ch

We establish sharp upper and lower bounds for the number of rational points of bounded anticanonical height on a smooth bihomogeneous threefold defined over $\mathbb{Q}$ and of bidegree $(1,2)$. These bounds are in agreement with Manin's conjecture.

\section{Introduction}

Let $n \geq 2$ and $d \geq 1$ be two integers such that $n \geq d$. Let $V_{d}^{n} \subset \mathbb{P}^{n} \times \mathbb{P}^{n}$ be the smooth hypersurface defined over a number field $K$ by the equation

$$
x_{0} y_{0}^{d}+\cdots+x_{n} y_{n}^{d}=0
$$

where we use the notation $(\mathbf{x}, \mathbf{y})=\left(\left(x_{0}: \cdots: x_{n}\right),\left(y_{0}: \cdots: y_{n}\right)\right)$ to denote the coordinates in the biprojective space $\mathbb{P}^{n} \times \mathbb{P}^{n}$.

The family of smooth bihomogeneous varieties $V_{d}^{n}$ is an excellent testing ground for the validity of Manin's conjecture on the asymptotic behavior of the number of rational points of bounded anticanonical height on Fano varieties (see [4]). For instance, Batyrev and Tschinkel have provided a famous counterexample to this conjecture in the case $n=3, d=3$, and under the assumption that $K$ contains a nontrivial cube root of unity. 
From now on, we focus on the case $K=\mathbb{Q}$. We define the usual exponential height function $H: \mathbb{P}^{n}(\mathbb{Q}) \rightarrow \mathbb{R}_{>0}$ as follows. Given $\mathbf{z} \in \mathbb{P}^{n}(\mathbb{Q})$, we can choose coordinates $\left(z_{0}: \cdots\right.$ : $\left.z_{n}\right)$ satisfying $\left(z_{0}, \ldots, z_{n}\right) \in \mathbb{Z}^{n+1}$ and $\operatorname{gcd}\left(z_{0}, \ldots, z_{n}\right)=1$, and then we can set

$$
H(\mathbf{z})=\max \left\{\left|z_{i}\right|, i=0, \ldots, n\right\}
$$

With this in mind, we can define a height function $\mathbf{H}: \mathbb{P}^{n}(\mathbb{Q}) \times \mathbb{P}^{n}(\mathbb{Q}) \rightarrow \mathbb{R}_{>0}$ by setting

$$
\mathbf{H}(\mathbf{x}, \mathbf{y})=H(\mathbf{x})^{n} H(\mathbf{y})^{n+1-d}
$$

for $(\mathbf{x}, \mathbf{y}) \in \mathbb{P}^{n}(\mathbb{Q}) \times \mathbb{P}^{n}(\mathbb{Q})$. For any Zariski open subset $U_{d}^{n}$ of $V_{d}^{n}$, we can introduce the number of rational points of bounded anticanonical height on $U_{d}^{n}$, that is

$$
N_{U_{d}^{n}, \mathbf{H}}(B)=\#\left\{(\mathbf{x}, \mathbf{y}) \in U_{d}^{n}(\mathbb{Q}), \mathbf{H}(\mathbf{x}, \mathbf{y}) \leq B\right\} .
$$

In this setting, Manin's conjecture predicts that there should exist an open subset $U_{d}^{n}$ of $V_{d}^{n}$ such that

$$
N_{U_{d}^{n}, \mathrm{H}}(B)=c B \log B(1+o(1))
$$

where $c>0$ is a constant depending on $V_{d}^{n}$ and $\mathbf{H}$, and which is expected to obey Peyre's prediction [7]. As already mentioned, this conjecture is known not to hold in such generality.

Let us mention that more generally, the term $\log B$ in conjecture (1.1) is expected to appear to the power $\rho-1$ where $\rho$ denotes the rank of the Picard group of the variety considered. A proof of the fact that the Picard group of $V_{d}^{n}$ is $\mathbb{Z}^{2}$ can be found in [10, Theorem 2.4].

The circle method is a traditional technique to count solutions to diophantine equations, and it has recently been applied by Schindler $[9,10]$ to count rational points on bihomogeneous varieties. In particular, [10, Theorem 1.2] states that smooth hypersurfaces in biprojective space $\mathbb{P}^{n_{1}} \times \mathbb{P}^{n_{2}}$ defined by general bihomogeneous forms of bidegree $\left(d_{1}, d_{2}\right)$ satisfy Manin's conjecture provided that $d_{1}, d_{2} \geq 2$ and $\min \left\{n_{1}, n_{2}\right\}>$ $3 \cdot 2^{d_{1}+d_{2}} d_{1} d_{2}$.

Similarly, the circle method is only expected to yield a proof of Manin's conjecture for $V_{d}^{n}$ if $n$ is exponentially large in terms of $d$.

It is natural to start by investigating the cases where $d$ is small. If $d=1$, then $V_{1}^{n}$ satisfies Manin's conjecture for any $n \geq 2$. This follows from the result of Franke et al. [4] on flag varieties, which makes use of the work of Langlands about the meromorphic continuation of Eisenstein series. Other proofs have then been obtained by many authors using a great variety of techniques (see $[2,8,11,12])$. 
The next case of interest is $d=2$. Here, there is nothing written down in the literature and, in particular, the cases $n=2$ and $n=3$ are known to be extremely hard problems. The aim of this article is to investigate what can be achieved in the case $n=2$.

Unfortunately, we are unable to establish Manin's conjecture for $V_{2}^{2}$. However, we are able to prove upper and lower bounds of the exact order of magnitude for $N_{U_{2}^{2}, \mathrm{H}}(B)$, where $U_{2}^{2}$ is the open subset defined by removing from $V_{2}^{2}$ the subset given by $x_{0} x_{1} x_{2} y_{0} y_{1} y_{2}=0$.

Our main result is the following theorem.

Theorem 1. We have the bounds

$$
B \log B \ll N_{U_{2}^{2}, \mathrm{H}}(B) \ll B \log B .
$$

It is worth emphasizing that these bounds are in agreement with the prediction of Manin (1.1).

Let us give a sketch of the proof. In what follows, we denote by $\varphi_{i}: \mathbb{P}^{2} \times \mathbb{P}^{2} \rightarrow \mathbb{P}^{2}$, $i \in\{1,2\}$, the two projections.

First, we remark that proving the lower bound is not hard. Indeed, it suffices to note that the contribution to $N_{U_{2}^{2}, \mathrm{H}}(B)$ of the fibers of $\varphi_{2}$ corresponding to rational points $y \in \mathbb{P}^{2}(\mathbb{Q})$ whose height is bounded by a small power of $B$ is of the expected order of magnitude. This is achieved in Section 3.

The proof of the upper bound is more intricate. It mainly relies on Lemma 4 which gives an upper bound for the number of solutions to a slightly more general equation than $x_{0} y_{0}^{2}+x_{1} y_{1}^{2}+x_{2} y_{2}^{2}=0$. To prove this lemma, we make use of both geometry of numbers and analytic number theory results.

More specifically, we get a first upper bound by estimating the number of $\mathbf{x} \in$ $\mathbb{P}^{2}(\mathbb{Q})$ for fixed $y \in \mathbb{P}^{2}(\mathbb{Q})$ and by summing trivially over the fibers of $\varphi_{2}$. Similarly, we obtain a second upper bound by estimating the number of $y \in \mathbb{P}^{2}(\mathbb{Q})$ for fixed $\mathbf{x} \in \mathbb{P}^{2}(\mathbb{Q})$. However, it is worth noting that the summation over the fibers of $\varphi_{1}$ has to be carried out nontrivially because we need to take advantage of the fact that most diagonal conics do not have a rational point. To complete the proof, it only remains to minimize these two upper bounds, basically depending on the respective sizes of $\mathbf{x}$ and $\mathbf{y}$.

Finally, it is worth mentioning that Lemma 4 will be useful in other settings. In particular, it plays a crucial role in the work of the author [6], where it is proved that the number of rational points of bounded height on certain elliptic fibrations grows linearly, as predicted by Manin's conjecture. 


\section{Geometry of Numbers}

We now recall two lemmas that provide upper bounds for the number of solutions to certain homogeneous diagonal equations in three variables and constrained in boxes. The first of these two lemmas is concerned with the case of a linear equation and is due to Heath-Brown [5, Lemma 3].

Lemma 1. Let $\mathbf{w}=\left(w_{0}, w_{1}, w_{2}\right) \in \mathbb{Z}^{3}$ be a primitive vector and let $U_{i} \geq 1$ for $i \in\{0,1,2\}$. Let also $N_{\mathrm{w}}=N_{\mathrm{w}}\left(U_{0}, U_{1}, U_{2}\right)$ be the number of primitive vectors $\left(u_{0}, u_{1}, u_{2}\right) \in \mathbb{Z}^{3}$ satisfying $\left|u_{i}\right| \leq U_{i}$ for $i \in\{0,1,2\}$ and the equation

$$
u_{0} w_{0}+u_{1} w_{1}+u_{2} w_{2}=0
$$

We have the bound

$$
N_{\mathrm{w}} \leq 12 \pi \frac{U_{0} U_{1} U_{2}}{\max \left\{\left|w_{i}\right| U_{i}\right\}}+4
$$

where the maximum is taken over $i \in\{0,1,2\}$. In particular, if $\mathbf{w} \in \mathbb{Z}_{\neq 0}^{3}$, then

$$
N_{\mathrm{w}} \ll \frac{\left(U_{0} U_{1} U_{2}\right)^{2 / 3}}{\left|w_{0} w_{1} w_{2}\right|^{1 / 3}}+1
$$

The second lemma deals with the case of a quadratic equation and immediately follows from the result of Browning and Heath-Brown [3, Corollary 2].

Lemma 2. Let $\mathbf{u}=\left(u_{0}, u_{1}, u_{2}\right) \in \mathbb{Z}_{\neq 0}^{3}$ be a vector satisfying the conditions $\operatorname{gcd}\left(u_{i}, u_{j}\right)=1$ for $i, j \in\{0,1,2\}, i \neq j$, and let $V_{i} \geq 1$ for $i \in\{0,1,2\}$. Let also $N_{\mathbf{u}}=N_{\mathbf{u}}\left(V_{0}, V_{1}, V_{2}\right)$ be the number of primitive vectors $\left(v_{0}, v_{1}, v_{2}\right) \in \mathbb{Z}^{3}$ satisfying $\left|v_{i}\right| \leq V_{i}$ for $i \in\{0,1,2\}$ and the equation

$$
u_{0} v_{0}^{2}+u_{1} v_{1}^{2}+u_{2} v_{2}^{2}=0
$$

We have the bound

$$
N_{\mathrm{u}} \ll\left(\frac{V_{0} V_{1} V_{2}}{\left|u_{0} u_{1} u_{2}\right|}+1\right)^{1 / 3} \tau\left(\left|u_{0} u_{1} u_{2}\right|\right) .
$$

We also need to consider how often a diagonal quadratic equation has a nontrivial integral solution. For this, we recall the following lemma, which is a particular case of the nice result of Browning [1, Proposition 1]. Let us note that this result is deep and builds upon several powerful analytic number theory tools.

Lemma 3. Let $\mathbf{f}=\left(f_{0}, f_{1}, f_{2}\right) \in \mathbb{Z}_{\neq 0}^{3}$ be a primitive vector and let $U_{i} \geq 1$ for $i \in\{0,1,2\}$. Let also $\mathcal{T}_{\mathbf{f}}\left(U_{0}, U_{1}, U_{2}\right)$ be the set of $\mathbf{u}=\left(u_{0}, u_{1}, u_{2}\right) \in \mathbb{Z}_{\neq 0}^{3}$ satisfying $\left|u_{i}\right| \leq U_{i}$ for $i \in\{0,1,2\}$, and 
$\operatorname{gcd}\left(u_{i}, u_{j}\right)=1$ for $i, j \in\{0,1,2\}, i \neq j$, and such that the equation

$$
f_{0} u_{0} v_{0}^{2}+f_{1} u_{1} v_{1}^{2}+f_{2} u_{2} v_{2}^{2}=0
$$

has a solution $\left(v_{0}, v_{1}, v_{2}\right) \in \mathbb{Z}_{\neq 0}^{3}$ with $\operatorname{gcd}\left(v_{i}, v_{j}\right)=1$ for $i, j \in\{0,1,2\}, i \neq j$. Let $\varepsilon>0$ be fixed. We have the bound

$$
\sum_{\mathbf{u} \in \mathcal{T}_{\mathbf{f}}\left(U_{0}, U_{1}, U_{2}\right)} 2^{\omega\left(\left|u_{0} u_{1} u_{2}\right|\right)} \ll\left|f_{0} f_{1} f_{2}\right|^{\varepsilon} U_{0} U_{1} U_{2} M_{\varepsilon}\left(U_{0}, U_{1}, U_{2}\right),
$$

where

$$
M_{\varepsilon}\left(U_{0}, U_{1}, U_{2}\right)=1+\max _{\{i, j, k\}=\{0,1,2\}}\left(U_{i} U_{j}\right)^{-1 / 2+\varepsilon} \log 2 U_{k}
$$

These three lemmas together allow us to prove a sharp upper bound for the number of solutions $(\mathbf{u}, \mathbf{v}) \in \mathbb{Z}_{\neq 0}^{3} \times \mathbb{Z}_{\neq 0}^{3}$ to the equation of Lemma 3 and constrained in boxes. More precisely, we establish the following lemma, which is the key result in the proof of the upper bound in Theorem 1.

Lemma 4. Let $\mathbf{f}=\left(f_{0}, f_{1}, f_{2}\right) \in \mathbb{Z}_{\neq 0}^{3}$ be a vector satisfying the conditions $\operatorname{gcd}\left(f_{i}, f_{j}\right)=1$ for $i, j \in\{0,1,2\}, i \neq j$, and let $U_{i}, V_{i} \geq 1$ for $i \in\{0,1,2\}$. Let also $N_{\mathrm{f}}=N_{\mathrm{f}}\left(U_{0}, U_{1}, U_{2}, V_{0}, V_{1}, V_{2}\right)$ be the number of vectors $\left(u_{0}, u_{1}, u_{2}\right) \in \mathbb{Z}_{\neq 0}^{3}$ and $\left(v_{0}, v_{1}, v_{2}\right) \in \mathbb{Z}_{\neq 0}^{3}$ satisfying $\left|u_{i}\right| \leq U_{i},\left|v_{i}\right| \leq V_{i}$ for $i \in\{0,1,2\}$, and the equation

$$
f_{0} u_{0} v_{0}^{2}+f_{1} u_{1} v_{1}^{2}+f_{2} u_{2} v_{2}^{2}=0
$$

and such that $\operatorname{gcd}\left(u_{i} v_{i}, u_{j} v_{j}\right)=1$ for $i, j \in\{0,1,2\}, i \neq j$. Let $\varepsilon>0$ be fixed and recall the definition of $M_{\varepsilon}\left(U_{0}, U_{1}, U_{2}\right)$ given in Lemma 3. We have the bound

$$
N_{\mathrm{f}} \ll\left|f_{0} f_{1} f_{2}\right|^{\varepsilon}\left(U_{0} U_{1} U_{2}\right)^{2 / 3}\left(V_{0} V_{1} V_{2}\right)^{1 / 3} M_{\varepsilon}\left(U_{0}, U_{1}, U_{2}\right) .
$$

Proof. First, let us fix $\left(v_{0}, v_{1}, v_{2}\right) \in \mathbb{Z}_{\neq 0}^{3}$ and let us start by bounding the number of $\left(u_{0}, u_{1}, u_{2}\right) \in \mathbb{Z}_{\neq 0}^{3}$ satisfying the conditions stated in the lemma. Since $\operatorname{gcd}\left(f_{0} v_{0}^{2}, f_{1} v_{1}^{2}, f_{2} v_{2}^{2}\right)=1$, Lemma 1 gives

$$
N_{\mathrm{f}} \ll \sum_{\substack{\left|v_{i}\right| \leq V_{i} \\ i \in\{0,1,2\}}}\left(\frac{1}{\left|f_{0} f_{1} f_{2}\right|^{1 / 3}} \frac{\left(U_{0} U_{1} U_{2}\right)^{2 / 3}}{\left|v_{0} v_{1} v_{2}\right|^{2 / 3}}+1\right) .
$$

In particular, this gives us a first upper bound

$$
N_{\mathbf{f}} \ll\left(U_{0} U_{1} U_{2}\right)^{2 / 3}\left(V_{0} V_{1} V_{2}\right)^{1 / 3}+V_{0} V_{1} V_{2}
$$


In a similar fashion, let us fix $\left(u_{0}, u_{1}, u_{2}\right) \in \mathbb{Z}_{\neq 0}^{3}$ and let us start by bounding the number of $\left(v_{0}, v_{1}, v_{2}\right) \in \mathbb{Z}_{\neq 0}^{3}$ satisfying the conditions stated in the lemma. The equation

$$
f_{0} u_{0} v_{0}^{2}+f_{1} u_{1} v_{1}^{2}+f_{2} u_{2} v_{2}^{2}=0
$$

and the coprimality conditions $\operatorname{gcd}\left(f_{i}, f_{j}\right)=\operatorname{gcd}\left(u_{i} v_{i}, u_{j} v_{j}\right)=1$ for $i, j \in\{0,1,2\}, i \neq j$, imply that $\operatorname{gcd}\left(f_{i} u_{i}, f_{j} u_{j}\right)=1$ for $i, j \in\{0,1,2\}, i \neq j$. We can thus apply Lemma 2. Recalling the notation introduced in Lemma 3, we obtain

$$
N_{\mathbf{f}} \ll \sum_{\mathbf{u} \in \mathcal{T}_{\mathbf{f}}\left(U_{0}, U_{1}, U_{2}\right)}\left(\frac{1}{\left|f_{0} f_{1} f_{2}\right|^{1 / 3}} \frac{\left(V_{0} V_{1} V_{2}\right)^{1 / 3}}{\left|u_{0} u_{1} u_{2}\right|^{1 / 3}}+1\right) \tau\left(\left|f_{0} f_{1} f_{2} u_{0} u_{1} u_{2}\right|\right) .
$$

This implies in particular that

$$
N_{\mathbf{f}} \ll\left|f_{0} f_{1} f_{2}\right|^{\varepsilon} \sum_{\mathbf{u} \in \mathcal{T}_{\mathbf{f}}\left(U_{0}, U_{1}, U_{2}\right)}\left(\frac{\left(V_{0} V_{1} V_{2}\right)^{1 / 3}}{\left|u_{0} u_{1} u_{2}\right|^{1 / 3}}+1\right) \tau\left(\left|u_{0} u_{1} u_{2}\right|\right) .
$$

Let us write $u_{i}=z_{i}^{2} \ell_{i}$ with $z_{i} \in \mathbb{Z}_{>0}$ and $\left|\mu\left(\left|\ell_{i}\right|\right)\right|=1$ for $i \in\{0,1,2\}$, and let us set $\mathbf{l}=$ $\left(\ell_{0}, \ell_{1}, \ell_{2}\right), \mathbf{g}=\left(f_{0} z_{0}^{2}, f_{1} z_{1}^{2}, f_{2} z_{2}^{2}\right)$ and $L_{i}=U_{i} / z_{i}^{2}$ for $i \in\{0,1,2\}$. We have

$$
N_{\mathrm{f}} \ll\left|f_{0} f_{1} f_{2}\right|^{\varepsilon} \sum_{\substack{z_{i} \leq U_{i}^{1 / 2} \\ i \in\{0,1,2\}}}\left(z_{0} z_{1} z_{2}\right)^{\varepsilon} \sum_{\substack{1 \in \mathcal{T}_{\mathrm{g}}\left(L_{0}, L_{1}, L_{2}\right) \\\left|\mu\left(\left|\ell_{0} \ell_{1} \ell_{2}\right|\right)\right|=1}}\left(\frac{\left(V_{0} V_{1} V_{2}\right)^{1 / 3}}{\left(z_{0} Z_{1} z_{2}\right)^{2 / 3}\left|\ell_{0} \ell_{1} \ell_{2}\right|^{1 / 3}}+1\right) 2^{\omega\left(\left|\ell_{0} \ell_{1} \ell_{2}\right|\right)} .
$$

Note that we have used the fact that $\ell_{0} \ell_{1} \ell_{2}$ is squarefree to replace the arithmetic function $\tau$ by $2^{\omega}$. Let $\varepsilon>0$ be fixed. We note that $\mathbf{g}$ is primitive so we can use Lemma 3 . Thus, applying partial summation and Lemma 3, we get

$$
N_{\mathrm{f}} \ll\left|f_{0} f_{1} f_{2}\right|^{2 \varepsilon} \sum_{\substack{Z_{i} \leq U_{i}^{1 / 2} \\ i \in\{0,1,2\}}} \frac{\left(U_{0} U_{1} U_{2}\right)^{2 / 3}\left(V_{0} V_{1} V_{2}\right)^{1 / 3}+U_{0} U_{1} U_{2}}{\left(z_{0} Z_{1} Z_{2}\right)^{2-3 \varepsilon}} M_{\varepsilon}\left(L_{0}, L_{1}, L_{2}\right) .
$$

Using the trivial inequality

$$
M_{\varepsilon}\left(L_{0}, L_{1}, L_{2}\right) \leq 1+\sum_{\{i, j, k\}=\{0,1,2\}}\left(U_{i} U_{j}\right)^{-1 / 2+\varepsilon}\left(Z_{i} Z_{j}\right)^{1-2 \varepsilon} \log 2 U_{k}
$$

we obtain

$$
\sum_{\substack{z_{i} \leq U_{i}^{1 / 2} \\ i \in\{0,1,2\}}} \frac{M_{\varepsilon}\left(L_{0}, L_{1}, L_{2}\right)}{\left(z_{0} Z_{1} Z_{2}\right)^{2-3 \varepsilon}} \ll M_{2 \varepsilon}\left(U_{0}, U_{1}, U_{2}\right)
$$

This finally gives us a second upper bound

$$
N_{\mathbf{f}} \ll\left|f_{0} f_{1} f_{2}\right|^{2 \varepsilon}\left(\left(U_{0} U_{1} U_{2}\right)^{2 / 3}\left(V_{0} V_{1} V_{2}\right)^{1 / 3}+U_{0} U_{1} U_{2}\right) M_{2 \varepsilon}\left(U_{0}, U_{1}, U_{2}\right) .
$$


As a result, putting together the upper bounds (2.1) and (2.2), and rescaling $\varepsilon$, we find in particular that

$$
N_{\mathrm{f}} \ll\left|f_{0} f_{1} f_{2}\right|^{\varepsilon}\left(\left(U_{0} U_{1} U_{2}\right)^{2 / 3}\left(V_{0} V_{1} V_{2}\right)^{1 / 3}+\min \left\{U_{0} U_{1} U_{2}, V_{0} V_{1} V_{2}\right\}\right) M_{\varepsilon}\left(U_{0}, U_{1}, U_{2}\right)
$$

The simple observation that

$$
\min \left\{U_{0} U_{1} U_{2}, V_{0} V_{1} V_{2}\right\} \leq\left(U_{0} U_{1} U_{2}\right)^{2 / 3}\left(V_{0} V_{1} V_{2}\right)^{1 / 3}
$$

completes the proof.

\section{The Lower Bound}

This section is devoted to the proof of the lower bound in Theorem 1. As stated in Section 1 , the proof merely draws upon the fact that the contribution to $N_{U_{2}^{2}, \mathrm{H}}(B)$ of the $y \in \mathbb{P}^{2}(\mathbb{Q})$ whose height is bounded by a small power of $B$ is already of the expected order of magnitude.

By definition of $N_{U_{2}^{2}, \mathrm{H}}(B)$, we have

$$
N_{U_{2}^{2}, \mathrm{H}}(B)=2 \#\left\{(\mathbf{x}, \mathbf{y}) \in \mathbb{Z}_{\neq 0}^{3} \times \mathbb{Z}_{>0}^{3}, \begin{array}{c}
x_{0} y_{0}^{2}+x_{1} y_{1}^{2}+x_{2} y_{2}^{2}=0 \\
\operatorname{gcd}\left(x_{0}, x_{1}, x_{2}\right)=\operatorname{gcd}\left(y_{0}, y_{1}, y_{2}\right)=1 \\
\max _{i, j \in\{0,1,2\}} x_{i}^{2} y_{j} \leq B
\end{array}\right\} .
$$

It is convenient to note that we thus have

$$
N_{U_{2}^{2}, \mathrm{H}}(B) \geq 12 \sum_{\substack{\mathrm{y} \in \mathbb{Z}_{>0}^{3} \\
\operatorname{gcd}\left(y_{0}, Y_{2}\right)=1 \\
y_{0}<y_{1}<y_{2} \leq B^{1 / 6}}} \#\left\{\mathbf{x} \in \mathbb{Z}_{\neq 0}^{3}, \begin{array}{c}
x_{0} y_{0}^{2}+x_{1} y_{1}^{2}+x_{2} y_{2}^{2}=0 \\
\max _{i \in\{0,1,2\}} x_{i}^{2} y_{2} \leq B
\end{array}\right\} .
$$

Since the conditions $\max _{i \in\{0,1\}} x_{i}^{2} y_{2} \leq B / 4$ and $y_{0}, y_{1}<y_{2}$ together with the equation $x_{0} y_{0}^{2}+x_{1} y_{1}^{2}+x_{2} y_{2}^{2}=0$ imply that $\max _{i \in\{0,1,2\}} x_{i}^{2} y_{2} \leq B$, we have

$$
N_{U_{2}^{2}, \mathbf{H}}(B) \geq 12 \sum_{\substack{\mathbf{y} \in \mathbb{Z}_{>0}^{3} \\
\operatorname{gcd}\left(y_{0}, Y_{2}\right)=1 \\
y_{0}<y_{1}<y_{2} \leq B^{1 / 6}}} \#\left\{\mathbf{x} \in \mathbb{Z}_{\neq 0}^{3}, \begin{array}{c}
x_{0} y_{0}^{2}+x_{1} y_{1}^{2}+x_{2} y_{2}^{2}=0 \\
\operatorname{gcd}\left(x_{0}, x_{1}, x_{2}\right)=1 \\
\max _{i \in\{0,1\}} x_{i}^{2} y_{2} \leq B / 4
\end{array}\right\}
$$


We can now remove the coprimality condition $\operatorname{gcd}\left(x_{0}, x_{1}, x_{2}\right)=1$ using a Möbius inversion. We get

$$
N_{U_{2}^{2}, \mathbf{H}}(B) \geq 12 \sum_{\substack{\mathbf{y} \in \mathbb{Z}_{>0}^{3} \\ \operatorname{gcd}\left(y_{0}, Y_{2}\right)=1 \\ y_{0}<Y_{1}<y_{2} \leq B^{1 / 6}}} \sum_{k \leq B^{1 / 2}} \mu(k) \mathcal{S}_{k}(\mathbf{y} ; B)
$$

where

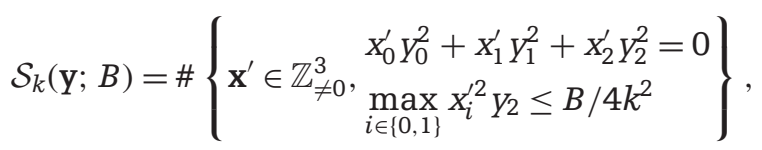

and where we have used the obvious notation $\mathbf{x}^{\prime}=\left(x_{0}^{\prime}, x_{1}^{\prime}, x_{2}^{\prime}\right)$. We now observe that

$$
\mathcal{S}_{k}(\mathbf{y} ; B)=\#\left\{\left(x_{0}^{\prime}, x_{1}^{\prime}\right) \in \mathbb{Z}_{\neq 0}^{2}, \begin{array}{l}
x_{0}^{\prime} y_{0}^{2}+x_{1}^{\prime} y_{1}^{2}=0\left(\bmod y_{2}^{2}\right) \\
\max _{i \in\{0,1\}} x_{i}^{\prime 2} y_{2} \leq B / 4 k^{2}
\end{array}\right\}+O\left(\frac{B^{1 / 2}}{k y_{2}^{1 / 2}}\right) .
$$

Since $\operatorname{gcd}\left(y_{0}, y_{2}\right)=1, y_{0}$ is invertible modulo $y_{2}^{2}$. Using the notation $y_{0}^{-1}$ to denote the inverse of $y_{0}$ modulo $y_{2}^{2}$, we have

$$
\begin{aligned}
\mathcal{S}_{k}(\mathbf{y} ; B) & =\sum_{\substack{x_{1}^{\prime} \in \mathbb{Z}_{\neq 0} \\
x_{1}^{2} y_{2} \leq B / 4 k^{2}}} \#\left\{x_{0}^{\prime} \in \mathbb{Z}_{\neq 0}, \begin{array}{l}
x_{0}^{\prime}=-y_{0}^{-2} x_{1}^{\prime} y_{1}^{2}\left(\bmod y_{2}^{2}\right) \\
x_{0}^{\prime 2} y_{2} \leq B / 4 k^{2}
\end{array}\right\}+O\left(\frac{B^{1 / 2}}{k y_{2}^{1 / 2}}\right) \\
& =\sum_{\substack{x_{1}^{\prime} \in \mathbb{Z}_{\neq 0} \\
x_{1}^{\prime 2} y_{2} \leq B / 4 k^{2}}}\left(\frac{B^{1 / 2}}{k y_{2}^{5 / 2}}+O(1)\right)+O\left(\frac{B^{1 / 2}}{k y_{2}^{1 / 2}}\right) \\
& =\frac{B}{k^{2} y_{2}^{3}}+O\left(\frac{B^{1 / 2}}{k y_{2}^{1 / 2}}\right) .
\end{aligned}
$$

Recalling the lower bound (3.1), we see that we have obtained

$$
N_{U_{2}^{2}, \mathrm{H}}(B) \geq 12 \sum_{\substack{\mathbf{y} \in \mathbb{Z}_{>0}^{3} \\ \operatorname{gcd}\left(y_{0}, Y_{2}\right)=1 \\ y_{0}<y_{1}<y_{2} \leq B^{1 / 6}}} \sum_{k \leq B^{1 / 2}} \mu(k)\left(\frac{B}{k^{2} y_{2}^{3}}+O\left(\frac{B^{1 / 2}}{k y_{2}^{1 / 2}}\right)\right) .
$$

This eventually gives

$$
N_{U_{2}^{2}, \mathrm{H}}(B) \gg B \log B
$$

which completes the proof of the lower bound in Theorem 1 . 


\section{The Upper Bound}

This section is concerned with establishing the upper bound in Theorem 1. As already explained in Section 1, the proof draws upon Lemma 4.

\subsection{Parametrization of the variables}

The following lemma provides us with a convenient parametrization of the rational points on $U_{2}^{2}$.

Lemma 5. Let $\mathcal{T}(B)$ be the number of $\left(f_{0}, f_{1}, f_{2}, g_{0}, g_{1}, g_{2}, h_{0}, h_{1}, h_{2}\right) \in \mathbb{Z}_{>0}^{9}$ and $\left(u_{0}, u_{1}, u_{2}, v_{0}, v_{1}, v_{2}\right) \in \mathbb{Z}_{\neq 0}^{6}$ satisfying the equation

$$
f_{0} u_{0} v_{0}^{2}+f_{1} u_{1} v_{1}^{2}+f_{2} u_{2} v_{2}^{2}=0
$$

and the conditions $\operatorname{gcd}\left(f_{i}, f_{j} g_{j} h_{i} u_{i} v_{j}\right)=\operatorname{gcd}\left(g_{i}, g_{j} h_{i} u_{i} v_{i} v_{j}\right)=\operatorname{gcd}\left(h_{i}, h_{j} v_{i}\right)=1 \quad$ and $\operatorname{gcd}\left(u_{i}, u_{j}\right)=\operatorname{gcd}\left(v_{i}, v_{j}\right)=1$ for $i, j \in\{0,1,2\}, i \neq j$, and the height conditions

$$
\left(\max _{\{i, j, k\}=\{0,1,2\}} f_{j} f_{k} g_{j}^{2} g_{k}^{2} h_{i}^{2}\left|u_{i}\right|\right)^{2}\left(\max _{\{i, j, k\}=\{0,1,2\}} f_{i} g_{i} h_{j} h_{k}\left|v_{i}\right|\right) \leq B .
$$

We have the equality

$$
N_{U_{2}^{2}, \mathrm{H}}(B)=\frac{1}{4} \mathcal{T}(B)
$$

Proof. We have

$$
N_{U_{2}^{2}, \mathrm{H}}(B)=\frac{1}{4} \#\left\{(\mathbf{x}, \mathbf{y}) \in \mathbb{Z}_{\neq 0}^{3} \times \mathbb{Z}_{\neq 0}^{3}, \begin{array}{c}
x_{0} y_{0}^{2}+x_{1} y_{1}^{2}+x_{2} y_{2}^{2}=0 \\
\operatorname{gcd}\left(x_{0}, x_{1}, x_{2}\right)=\operatorname{gcd}\left(y_{0}, y_{1}, y_{2}\right)=1 \\
\max _{i, j \in\{0,1,2\}} x_{i}^{2}\left|y_{j}\right| \leq B
\end{array}\right\} .
$$

For $\{i, j, k\}=\{0,1,2\}$, let us set $h_{i}=\operatorname{gcd}\left(y_{j}, y_{k}\right)$ and let us write $y_{i}=h_{j} h_{k} y_{i}^{\prime}$. The equation

$$
x_{0} h_{1}^{2} h_{2}^{2} y_{0}^{2}+x_{1} h_{0}^{2} h_{2}^{2} y_{1}^{2}+x_{2} h_{0}^{2} h_{1}^{2} y_{2}^{2}=0
$$

implies that for $i \in\{0,1,2\}$, we have $h_{i}^{2} \mid x_{i}$ so that we can write $x_{i}=h_{i}^{2} x_{i}^{\prime}$. We thus obtain the equation

$$
x_{0}^{\prime} y_{0}^{2}+x_{1}^{\prime} y_{1}^{2}+x_{2}^{\prime} y_{2}^{2}=0
$$

For $\{i, j, k\}=\{0,1,2\}$, let us set $X_{i}=\operatorname{gcd}\left(x_{j}^{\prime}, x_{k}^{\prime}\right)$ and let us write $x_{i}^{\prime}=X_{j} X_{k} u_{i}$. We obtain

$$
X_{1} X_{2} u_{0} y_{0}^{2}+X_{0} X_{2} u_{1} y_{1}^{2}+X_{0} X_{1} u_{2} y_{2}^{2}=0
$$


so that, for $i \in\{0,1,2\}$, we have $X_{i} \mid y_{i}^{2}$. As a consequence, for $i \in\{0,1,2\}$, there is a unique way to write $X_{i}=f_{i} g_{i}^{2}$ and $y_{i}^{\prime}=f_{i} g_{i} v_{i}$ for $f_{i}, g_{i} \in \mathbb{Z}_{>0}$ with $\operatorname{gcd}\left(g_{i}, v_{i}\right)=1$. Therefore, we obtain the equation

$$
f_{0} u_{0} v_{0}^{2}+f_{1} u_{1} v_{1}^{2}+f_{2} u_{2} v_{2}^{2}=0
$$

and it is not hard to check that the variables satisfy the coprimality conditions listed in the statement of the lemma, which completes the proof.

\subsection{Proof of the upper bound}

First, we note that the coprimality conditions $\operatorname{gcd}\left(f_{i}, v_{j}\right)=\operatorname{gcd}\left(u_{i}, u_{j}\right)=\operatorname{gcd}\left(v_{i}, v_{j}\right)=1$ for $i, j \in\{0,1,2\}, i \neq j$, and the equation

$$
f_{0} u_{0} v_{0}^{2}+f_{1} u_{1} v_{1}^{2}+f_{2} u_{2} v_{2}^{2}=0
$$

imply that we actually have $\operatorname{gcd}\left(u_{i} v_{i}, u_{j} v_{j}\right)=1$ for $i, j \in\{0,1,2\}, i \neq j$.

For $i \in\{0,1,2\}$, let $F_{i}, G_{i}, H_{i}, U_{i}, V_{i} \geq 1 / 2$ run over powers of 2 and let $\mathcal{M}$ be the number of $\left(f_{0}, f_{1}, f_{2}, g_{0}, g_{1}, g_{2}, h_{0}, h_{1}, h_{2}\right) \in \mathbb{Z}_{>0}^{9}$ and $\left(u_{0}, u_{1}, u_{2}, v_{0}, v_{1}, v_{2}\right) \in \mathbb{Z}_{\neq 0}^{6}$ satisfying the equation

$$
f_{0} u_{0} v_{0}^{2}+f_{1} u_{1} v_{1}^{2}+f_{2} u_{2} v_{2}^{2}=0
$$

the conditions $F_{i}<f_{i} \leq 2 F_{i}, G_{i}<g_{i} \leq 2 G_{i}, H_{i}<h_{i} \leq 2 H_{i}, U_{i}<\left|u_{i}\right| \leq 2 U_{i}$ and $V_{i}<\left|v_{i}\right| \leq 2 V_{i}$, and $\operatorname{gcd}\left(f_{i}, f_{j}\right)=\operatorname{gcd}\left(u_{i} v_{i}, u_{j} v_{j}\right)=1$ for $i, j \in\{0,1,2\}, i \neq j$. By Lemma 5 , we have

$$
N_{U_{2}^{2}, \mathbf{H}}(B) \ll \sum_{\substack{F_{i}, G_{i}, H_{i}, U_{i}, V_{i} \\ i \in\{0,1,2\}}} \mathcal{M}
$$

where the sum is taken over the $F_{i}, G_{i}, H_{i}, U_{i}, V_{i}, i \in\{0,1,2\}$, satisfying

$$
\left(\max _{\{i, j, k\}=\{0,1,2\}} F_{j} F_{k} G_{j}^{2} G_{k}^{2} H_{i}^{2} U_{i}\right)^{2}\left(\max _{\{i, j, k\}=\{0,1,2\}} F_{i} G_{i} H_{j} H_{k} V_{i}\right) \leq B .
$$

By choosing $\varepsilon=1 / 6$ in Lemma 4 , we get

$$
\mathcal{M} \ll\left(F_{0} F_{1} F_{2}\right)^{7 / 6} G_{0} G_{1} G_{2} H_{0} H_{1} H_{2}\left(U_{0} U_{1} U_{2}\right)^{2 / 3}\left(V_{0} V_{1} V_{2}\right)^{1 / 3} M_{1 / 6}\left(U_{0}, U_{1}, U_{2}\right)
$$

Recalling the definition of $M_{1 / 6}\left(U_{0}, U_{1}, U_{2}\right)$ given in Lemma 3, we define

$$
\mathcal{M}_{1}=\left(F_{0} F_{1} F_{2}\right)^{7 / 6} G_{0} G_{1} G_{2} H_{0} H_{1} H_{2}\left(U_{0} U_{1} U_{2}\right)^{2 / 3}\left(V_{0} V_{1} V_{2}\right)^{1 / 3},
$$


and

$$
\begin{aligned}
\mathcal{M}_{2}= & (\log B)\left(F_{0} F_{1} F_{2}\right)^{7 / 6} G_{0} G_{1} G_{2} H_{0} H_{1} H_{2}\left(U_{0} U_{1} U_{2}\right)^{2 / 3}\left(V_{0} V_{1} V_{2}\right)^{1 / 3} \\
& \times\left(\min _{i, j \in\{0,1,2\}, i \neq j} U_{i} U_{j}\right)^{-1 / 3},
\end{aligned}
$$

and also

$$
\mathcal{N}_{\ell}(B)=\sum_{\substack{F_{i}, G_{i}, H_{i}, U_{i}, V_{i} \\ i \in\{0,1,2\}}} \mathcal{M}_{\ell}
$$

for $\ell \in\{1,2\}$, and where the sum is taken over the $F_{i}, G_{i}, H_{i}, U_{i}, V_{i}, i \in\{0,1,2\}$, satisfying the conditions (4.1). We thus have

$$
N_{U_{2}^{2}, \mathrm{H}}(B) \ll \mathcal{N}_{1}(B)+\mathcal{N}_{2}(B) .
$$

Let us start by taking care of $\mathcal{N}_{1}(B)$. For this, let us sum over $V_{0}, V_{1}$, and $V_{2}$ using the conditions (4.1). We obtain

$$
\begin{aligned}
\mathcal{N}_{1}(B) \ll & B \sum_{\substack{F_{i}, G_{i}, H_{i}, U_{i} \\
i \in\{0,1,2\}}}\left(F_{0} F_{1} F_{2}\right)^{5 / 6}\left(G_{0} G_{1} G_{2}\right)^{2 / 3}\left(H_{0} H_{1} H_{2}\right)^{1 / 3}\left(U_{0} U_{1} U_{2}\right)^{2 / 3} \\
& \times\left(\max _{\substack{\{i, j, k\}=\{0,1,2\} \\
F_{j}}} F_{k} G_{j}^{2} G_{k}^{2} H_{i}^{2} U_{i}\right)^{-2} .
\end{aligned}
$$

By symmetry, we can assume that

$$
\max \left\{F_{0} F_{2} G_{0}^{2} G_{2}^{2} H_{1}^{2} U_{1}, F_{0} F_{1} G_{0}^{2} G_{1}^{2} H_{2}^{2} U_{2}\right\} \leq F_{1} F_{2} G_{1}^{2} G_{2}^{2} H_{0}^{2} U_{0}
$$

Let us sum over $U_{1}$ and $U_{2}$ using the inequalities (4.3). We obtain

$$
\mathcal{N}_{1}(B) \ll B \sum_{\substack{F_{i}, G_{i}, H_{i}, U_{0} \\ i \in\{0,1,2\}}}\left(F_{0} F_{1} F_{2}\right)^{-1 / 2}\left(G_{0} G_{1} G_{2}\right)^{-2}\left(H_{0} H_{1} H_{2}\right)^{-1},
$$

which finally gives

$$
\mathcal{N}_{1}(B) \ll B \log B
$$

Let us now deal with $\mathcal{N}_{2}(B)$. We can assume by symmetry that

$$
\min _{i, j \in\{0,1,2\}, i \neq j} U_{i} U_{j}=U_{1} U_{2}
$$

We thus have

$$
\mathcal{M}_{2} \ll(\log B)\left(F_{0} F_{1} F_{2}\right)^{7 / 6} G_{0} G_{1} G_{2} H_{0} H_{1} H_{2} U_{0}^{2 / 3}\left(U_{1} U_{2}\right)^{1 / 3}\left(V_{0} V_{1} V_{2}\right)^{1 / 3}
$$


Once again, let us sum over $V_{0}, V_{1}$, and $V_{2}$ using the conditions (4.1). We find that

$$
\begin{aligned}
\mathcal{N}_{2}(B) \ll & B(\log B) \sum_{\substack{F_{i}, G_{i}, H_{i}, U_{i} \\
i \in\{0,1,2\}}}\left(F_{0} F_{1} F_{2}\right)^{5 / 6}\left(G_{0} G_{1} G_{2}\right)^{2 / 3}\left(H_{0} H_{1} H_{2}\right)^{1 / 3} U_{0}^{2 / 3}\left(U_{1} U_{2}\right)^{1 / 3} \\
& \times\left(\max _{\substack{\{, j, k\}=\{0,1,2\} \\
F_{j}}} F_{j} F_{k} G_{j}^{2} G_{k}^{2} H_{i}^{2} U_{i}\right)^{-2} .
\end{aligned}
$$

Now, let us use the inequality

$$
\left(\max _{\{i, j, k\}=\{0,1,2\}} F_{j} F_{k} G_{j}^{2} G_{k}^{2} H_{i}^{2} U_{i}\right)^{2} \geq F_{0}\left(F_{1} F_{2}\right)^{3 / 2} G_{0}^{2}\left(G_{1} G_{2}\right)^{3} H_{0}^{2} H_{1} H_{2} U_{0}\left(U_{1} U_{2}\right)^{1 / 2} .
$$

This gives us

$$
\mathcal{N}_{2}(B) \ll B(\log B) \sum_{\substack{F_{i}, G_{i}, H_{i}, U_{i} \\ i \in\{0,1,2\}}}\left(F_{0}\left(F_{1} F_{2}\right)^{4} G_{0}^{8}\left(G_{1} G_{2}\right)^{14} H_{0}^{10}\left(H_{1} H_{2}\right)^{4} U_{0}^{2} U_{1} U_{2}\right)^{-1 / 6},
$$

and therefore, we obtain

$$
\mathcal{N}_{2}(B) \ll B \log B
$$

Putting together the three upper bounds (4.2), (4.4), and (4.5) completes the proof of the upper bound in Theorem 1.

\section{Acknowledgements}

It is a pleasure for the author to thank Tim Browning for interesting explanations and comments on the results given in Section 2, and Damaris Schindler for kindly answering questions about her works, and for comments on an earlier draft of this paper. The author would also like to thank the referee for his very careful work.

\section{Funding}

The financial support and the perfect working conditions provided by the Institute for Advanced Study are gratefully acknowledged. This material is based upon work supported by the National Science Foundation under agreement No. DMS-1128155. Any opinions, findings and conclusions or recommendations expressed in this material are those of the author and do not necessarily reflect the views of the National Science Foundation.

\section{References}

[1] Browning, T. D. "The density of rational points on a certain singular cubic surface." Journal of Number Theory 119, no. 2 (2006): 242-83. 
[2] Browning, T. D. "The divisor problem for binary cubic forms." Journal de Théorie des Nombres de Bordeaux 23, no. 3 (2011): 579-602.

[3] Browning, T. D. and D. R. Heath-Brown. "Counting rational points on hypersurfaces." Journal für die Reine und Angewandte Mathematik 584 (2005): 83-115.

[4] Franke, J., Y. I. Manin, and Y. Tschinkel. "Rational points of bounded height on Fano varieties." Inventiones Mathematicae 95, no. 2 (1989): 421-35.

[5] Heath-Brown, D. R. "Diophantine approximation with square-free numbers." Mathematische Zeitschrift 187, no. 3 (1984): 335-44.

[6] Le Boudec, P. "Linear growth for certain elliptic fibrations." International Mathematics Research Notices 2015, no. 21 (2015): 10859-71.

[7] Peyre, E. "Hauteurs et mesures de Tamagawa sur les variétés de Fano." Duke Mathematical Journal 79, no. 1 (1995): 101-218.

[8] Robbiani, M. “On the number of rational points of bounded height on smooth bilinear hypersurfaces in biprojective space." Journal of the London Mathematical Society (2) 63, no. 1 (2001): 33-51.

[9] Schindler, D. "Bihomogeneous forms in many variables." J. Théor. Nombres Bordeaux (2013): preprint. arXiv:1301.6516v1.

[10] Schindler, D. "Manin's conjecture for certain biprojective hypersurfaces." Journal für die Reine und Angewandte Mathematik (2013): preprint. arXiv:1307.7069v1.

[11] Spencer, C. V. "The Manin conjecture for $x_{0} y_{0}+\cdots+x_{s} y_{s}=0$." Journal of Number Theory 129, no. 6 (2009): 1505-21.

[12] Thunder, J. L. "Asymptotic estimates for rational points of bounded height on flag varieties." Compositio Mathematica 88, no. 2 (1993): 155-86. 